\title{
Teoría y práctica del aseguramiento privado de salud en Chile: brechas, consistenciay reforma pendiente.
}

\section{Teoria e Prática de seguros privados de saúde no Chile: lacunas, coerência e reforma pendente.}

\section{Theory and Practice of private health insurance in Chile: gaps, consistence and pending reform.}

Cesar Humberto GATTINI ${ }^{1}$

Oscar ARTEAGA ${ }^{2}$

RESUMEN: Este artículo revisa el rolque cumplen los seguros privados de salud en Chile, comparado con los principios y recomendaciones internacionales sobre seguridad social en salud. El objetivo fue revisar la situación actual desdela perspectiva de salud pública,los sistemas de salud,protección social, y los derechos y necesidades de la población.Creados en 1981, estos seguros han logrado un sólido desarrolloinstitucional, pero mantienen problemas e inconsistencias que limitan el acceso adecuado al aseguramiento y alos beneficios requeridos delsistema de salud. Sus limitaciones se relacionan con el modeloneoliberal prevalente enun mercado poco regulado;suclientelay rentabilidad está concentradaen cotizantes de mayores ingresos ymenor riesgo, ante quienes realizan fijación arbitraria de planes y alzas de precios. En su quehacer, predominael interés comercial y sustentabilidad económica por sobrelas necesidades y capacidad de pagode sus beneficiarios. Sin embargo, continúan siendo la alternativa preferente de elección en el grupo de población con mayores ingresos, debido a la limitada y no competitiva opciónque les puede ofrecer el seguro público.Si bien estánconsolidados, sonvulnerables a cambios que puedan arriesgar su sustentabilidad y rentabilidad. Se les harealizado mejorías parcialesy ajustesdesde 1990, lo queaún esinsuficiente. Desde 2010, existen estudios y propuestas gubernamentales sobre reformas concretas, loque se mantiene pendiente, debido a las restricciones que impone el modelo prevalente y la presión de los grupos de interés económico. Palabras clave: Política de Salud; Seguro de Salud; Sistema de Salud; Reforma de salud; Chile

RESUMO: Este artigo analisa o papel desempenhado pelo seguro de saúde privado no Chile, em

1 Escuela de Salud Pública Universidad de Chile.E-mail: cesargattini@hotmail.com

2 Escuela de Salud Pública F. de Medicina Universidad de Chile. E-mail: oarteaga@med.uchile.cl 
contraste com os princípios e recomendações internacionais para a segurança social de saúde. O objetivo foi realizar a avaliação a partir da perspectiva da saúde pública, sistemas de saúde, a proteção social e os direitos e necessidades da população. Criado em 1981, essas seguradoras alcançaram desenvolvimento institucional sólido, mas continuam a ter problemas e inconsistências que limitam o direito de acesso às prestações de seguros e sistema de saúde necessários. Suas limitações estão relacionadas com o modelo neoliberal vigente em um mercado em grande parte não regulamentada; seus clientes e lucratividade está concentrada nas contribuintes de renda mais alta e menor risco, para aqueles queestabelecem planos arbitrários e definem aumentos de preços. Em seu trabalho, prevalece o interesse comercial ea sustentabilidade econômica sobre as necessidades e capacidade de pagamento dos beneficiários. No entanto, eles continuam a ser a alternativa preferida de escolha no grupo de população com maior renda, devido à opção limitada e não-competitivo que pode oferecer seguro público. Se eles estão bem estabelecidas, eles são vulneráveis a mudanças que podem pôr em risco a sua sustentabilidade e rentabilidade. Ele passou por melhorias parciais e ajustes desde 1990, o que ainda é insuficiente. Desde 2010, há estudos e propostas do governo para reformas concretas, que ainda está pendente, devido às restrições impostas pelo modelo vigente e pressão de grupos de interesse econômico. Palavras-chave: Política de Saúde; Plano de saúde; Sistema de Saúde; A reforma dos cuidados de saúde; Chile.

SUMMARY: This article reviews the role of the private health insurance in Chile, contrasted with the principles and recommendations on social security health. The objective was to conduct that review from the perspective of public health, health systems, social protection, as well as the population's rights and needs. Created in 1981, those insurers have achieved solid institutional development, but problems and inconsistencies remain, limitingadequate access to insurance benefits and to the health system. Their limitations are related to the prevailing neoliberal model within a largely unregulated market; their customers and profitability are concentrated in higherincome contributors and lower risk, whilst the insurersset arbitrary plans and price increase. Commercial interest and economic sustainability prevail over the beneficiaries' needs and ability to pay. However, they remain as the preferred alternative of choice in the highest income population group, due to the limited and non-competitive option that the public insurance can offer. They are well established but vulnerable to changes that may endanger their sustainability and profitability. They have received partial improvements and adjustments since 1990, which is still insufficient. Since 2010, there are studies and government proposals for concrete reforms, what it is still pending, due to restrictions imposed by the prevailing model and the pressure from economic interest groups. Keywords: Health Policy; Health insurance; Health System; Health Care Reform; Chile

\section{INTRODUCCIÓN}

En cada país, existen distintos modos de estructurar y administrar el sistema de salud, incluyendo los mecanismos de financiamiento y aseguramiento que son posibles de establecer y administrar. Su desarrollo depende de las posibilidades y limitaciones existentes, y se orienta por las experiencias, 


\section{$31 / /$}

valores e ideologías predominantes. ${ }^{1-3}$

Los seguros de salud contribuyen al financiamiento de un sistema de salud, favoreciendo la movilización, acumulación y asignación de recursos para cubrir las necesidades de salud de las personas, individual o colectivamente, en el sistema de salud. ${ }^{2}$ Los seguros de salud forman parte de la protección social en salud, la que a su vez es entendida como

"La garantía de cobertura que la sociedad otorga, a través de los poderes públicos, para que todos los individuos, o grupos de individuos, puedan satisfacer sus demandas o necesidades de salud a través del acceso a alguno de los subsistemas de salud en condiciones adecuadas de calidad, oportunidad y dignidad, sin que la capacidad de pago sea un factor restrictivo". 4

Los seguros de salud contribuyen a lograr la cobertura universal de salud y contribuir a la satisfacción de necesidades de todos los habitantes de un país, en términos de derechos, necesidades y equidad, así como de protección social global. ${ }^{2-6}$

Chile, al igual que otros países de Sudamérica fue sujeto a radicales reformas políticas y económicas nacionales, lo que incluyó al sector salud. Bajo un régimen autoritario y enfoque económico neoliberal, desde la década de los años setenta se favoreció la privatización, la eficiencia competitiva y la reducción del rol de Estado, lo que tuvo impacto negativo en la protección social y aumentó lainequidad en el sistema de salud. Dicha experiencia fue indicada como modelo por el Banco Mundia. ${ }^{1,6-9}$

Después de un largo desarrollo de protección global bajo distintos modelos parciales y cuatro décadas de aplicación del modelo actual, Chile ya cuenta con experiencia y lecciones en el tema de seguros públicos y privados de salud, que le permitan conocer su situación y necesidades de perfeccionamiento. Según estudios y propuestas de cambio, y en línea con las recomendaciones internacionales, es posible aplicar agendas de acción correctivas que permitan que los seguros de salud puedan cumplan su objetivo en favor de la salud de la población. En el caso de seguros privados, sus objetivos incluyen constituir empresas que junto a funcionar con adecuada solvencia y reglas de mercado, contribuyan a la protección social en materias de salud, según derechos y necesidades de sus beneficiarios. ${ }^{1,4-6,10-11}$ En 2010, OMS recomendó tres tipos de medidas necesarias: Pagar por la salud mediante fórmulas que no impidan el acceso a los servicios; consolidar los fondos mancomunados y adoptar el sistema de pago obligatorio; y utilizar los recursos de manera más eficiente y redistributiva. ${ }^{2}$

En el caso chileno, es deber del Estado responder al mandato constitucional conforme al cual debe garantizar el derecho a la protección de la salud. En este contexto y dadas las limitaciones y discriminación existentes con los seguros privados, el gobierno chileno decidió en 2014 la conformación de la Comisión Asesora Presidencial para redactar una propuesta de proyecto de ley para realizar una reforma a este sistema, que estuviera en "el contexto del funcionamiento global 
del sistema de seguridad social en el ámbito de la salud...". ${ }^{12}$

Junto a conocer las disparidades o brechas entre lo planteado desde el punto de vista teórico y lo real, es importante estudiar sus causas. ${ }^{13}$ Ante la necesidad de cambios, también es importante identificar las dificultades que impiden que los seguros de salud puedan cumplir sus objetivos y requisitos de buena práctica, que y disminuyenla factibilidad de lograr las condiciones políticas, legales y económicas paraque los seguros cumplan adecuadamente su función.En la revisión del presente trabajo, se ha considerado los elementos clave del análisis de la financiación sanitaria que plantea la OMS (2010) ${ }^{2}$ y de las bases para diseñar las etapas principales de financiación de la salud, que están centradas en la recaudación de fondos suficientes, su agrupación, y su utilización de modo inteligente, para garantizar que su disponibilidad y equidad. ${ }^{2-6}$

El objetivo de esta revisión fue comparar el rol observado de los seguros privados de salud en Chile, con lo esperado respecto a su función y aportes, bajo una doble perspectiva: (a) como empresas administradoras de seguros que contribuyen al adecuado financiamiento del sistema de salud y de su acceso, y (b) como entidades de la protección social que gestionan recursos para contribuir a la eficiencia y efectividad del sistema de salud en Chile, y a la mejor satisfacción de necesidades de salud de la población y bajo la perspectiva de solidaridad. ${ }^{2-5}$

En la revisión realizada, también se ha considerado el marco de siete requisitos que debe cumplir el aseguramiento de salud,definido por la Comisión Asesora Presidencial para el Estudio y Propuesta de un Nuevo Marco Jurídico para el Sistema Privado de Salud (Chile, 2014) ${ }^{12}$, en el contexto de la solidaridad valorada como un principio básico:

- Universalidad del sistema: todos y todas tienen los mismos derechos en materia de salud.

- Financiamiento solidario del sistema.

- Prestaciones amplias y universales, de carácter integral, incluido la atención primaria, como modelo de trabajo sanitario y no sólo como puerta de entrada al sistema.

- Mecanismos de compra que aseguren eficiencia y eficacia sanitaria.

- Afiliación abierta y no discriminatoria (libre elección de institución aseguradora).

- Seguro de largo plazo para todo el ciclo vital.

- Evaluación comunitaria de riesgos para la fijación de primas. ${ }^{12}$

Urriola (2006) ${ }^{10}$ plantea tres dimensiones que requiere cumplir la protección social de la salud: que la cobertura de población sea universal, que sus beneficiarios tengan cobertura garantizada de beneficios o prestaciones a los cuales tienen derecho, y que haya protección financiera para que los gastos no aumenten la desigualdad financiera o de salud. ${ }^{10}$ Una adecuada revisión del tema de 


\section{$33 / /$}

seguros de salud trasciende el ámbito específico económico o de la salud pública, pues serequiere de un análisis multidimensional del macro-contexto político, económico, social y sanitario del país, así como de la situación social, económica y sanitaria en que se encuentran los distintos grupos de habitantes del país. ${ }^{6,10-11}$

Al diseñar agendas de cambio, cabe considerar que entre las instituciones y grupos de interés, tiende a haber consenso respecto a los objetivos de los seguros de salud y las necesidades sanitarias de la población, pero se produce un disenso cuando surgen conflictos de interés, especialmente cuando se considera el mercado privado y los riesgos para la solvencia y sustentabilidad del aseguramiento privado de salud.En este contexto, cobra importancia revisar la factibilidad de realizar cambios y reformas de perfeccionamiento, y los obstáculos políticos, legales y económicos que impiden ese mejoramiento..$^{2,11-12}$

\section{ASPECTOS OBSERVADOS Y ESPERADOS DEL SEGURO PRIVADO DE SALUD}

\section{Características del seguro y sistema de salud}

El sistema de salud en Chile tiene una naturaleza mixta, con una mezcla público/privada tanto en la previsión como provisión de servicios; el rol rector y regulador es ejercido por el Estado a través del Ministerio de Salud y la Superintendencia de Salud (esta última supervisa y controla los seguros de salud). Las funciones de financiamiento y provisión de servicios se encuentran separadas. El financiamiento proviene principalmente del Estado, cotizaciones de trabajadores y empresas. La previsión de salud se logra con diversas entidades de seguros de salud del Sector público y privado. Hay múltiples entidades prestadoras de atención, tanto públicas como privadas, distribuidas a través del país. Gracias a esa amplia cobertura de previsión y prestadores de servicios asistenciales, la atención de salud alcanza una cobertura legal (vía aseguramiento o protección social) y geográfica que es prácticamente universal. ${ }^{10-14}$

El financiamiento del sistema mixto de salud incluye aportes públicos y privados, que se asignan y transfieren entre las distintas entidades de aseguramiento y provisión de servicios. El aporte público proviene del presupuesto general del Estado, basado en impuestos generales y específicos de las personas. El aporte privado incluye aportes directos e indirectos, por gastos de bolsillo de las personas y cotizaciones de los afiliados al sistema previsional privado. Los gastos de bolsillo corresponden a los copagos por atenciones médicas, pagos en medicamentos y el pago por atenciones médicas particulares que los usuarios efectúan directamente al prestador de servicios. En 2011, el gasto total en salud representaba 8\% del PIB. El 47,4 \% de ello es público y representa el 16\% del presupuesto del Gobierno. El 65\% del gasto privado es gasto directo o de bolsillo (que es el más regresivo). El gasto en salud per cápita ha aumentado anualmente, pero la proporción del gasto privado es mayor que la del público, lo que dificulta alcanzar universalidad con equidad y eficiencia en el sistema de salud. ${ }^{11-14}$ 
El aseguramiento público está a cargo del Fondo Nacional de Salud (FONASA) y el sistema privado es administrado por las Instituciones de Salud Previsional (ISAPRE). Estas en general están abiertas al mercado, aunque hay algunas cerradas (como es el caso de trabajadores del cobre). En 2011, FONASA cubría tres cuartos de la población (76\%, incluyendo también a las personas pobres) y las ISAPRE el 17\%. El 7\% estaba cubierto por otros sistemas específicos (como el de Fuerzas Armadas), y el restante 4\% no registraba aseguramiento formal. Existe un fondo de protección contra gastos catastróficos. Hay otras instituciones públicas y privadas que otorgan seguros más específicos, como accidentes escolares, laborales o de tránsito. Sin embargo, este artículo se refiere a las ISAPRE, como aseguradoras privadas que asumen el seguro global de salud de sus beneficiarios. En complemento de protección social, se cuenta con medidas como el Régimen de Garantías Explícitas (GES) que cubre el grupo de patologías garantizadas tanto para asegurados públicos como privados, y la iniciativa Chile Crece Contigo, que otorga protección social integral a niños menores de condición social vulnerable. ${ }^{10-14}$

Las características del sistema privado en Chile, tienen similitud con seguros de algunos otros países de Latinoamérica, en especial Colombia, y cobra importancia porque su existencia ha sido difundida internacionalmente como un exitoso y moderno sistema de previsión privado, pero no cuenta con evidencia que avale dicho éxito y su aplicación requiere ser adecuadamente evaluada, para evitar sesgos en difusión de modelos no respaldados plenamente por la práctica, sobre una experiencia que cuenta ya con más de tres décadas. ${ }^{8-9}$

\section{El modeloeconómico prevalente}

El sistema de seguros privados fue creado bajo reforma global y radical del Estado y de la economía. Desde su creación y aún cuando desde 1990 se cuenta en general con gobiernos de coalición de centro izquierda que han mejorado el gasto y protección social, se mantiene el enfoque neoliberal en la economía y el predominio del mercado sobre el Estado.Se mantiene el rol subsidiario del Estado, con fomento de privatización y reducción de tamaño y funciones estatales. La entrega de funciones públicas a seguros privados se basa en la libertad de elección de opciones públicas o privadas, para aseguramiento y atención de salud.La competencia es uno de los motores de la economía,pero esa competitividad es relativa en los seguros privados de salud, que funcionan en conjunto en defensa corporativa del grupo de ISAPRES, en torno a sus intereses comerciales comunes, y hay oligopolio (tres de ellas tienen alrededor de $80 \%$ de afiliados). ${ }^{9-11}$

Se mantiene el supuesto original de que el país está encaminado a un desarrollo industrializado y de crecimiento económico que pueda generar riqueza que a su vez llegue a la población general. Bajo esta expectativa, se continúa promoviendo elaumento de aseguramiento y atención privada de salud, mientras que el aseguramiento público es asumido como la principal opción para los más pobres.El sistema de la privatización de seguros de salud fue aplicado en base a los planteamientos teóricos de un modeloimpuesto en un período autoritario y bajo la influencia del Banco Mundial, y no se basó enpropuestas técnicas o económicas - de sustento científico - que pudieran haberse 


\section{$35 / /$}

respaldado deestudios económicos, sociales o de salud del país. ${ }^{3,11} \mathrm{El}$ sistema de seguros privados de salud en Chile ha sido publicitado internacionalmente como un ejemplo positivo de protección social, pero la sistemática evidencia no avala dicha afirmación. ${ }^{3,10-11}$ Surge entonces la necesidad de contar con investigaciones y evidencia que permita orientar políticas públicas que favorezcan mejor aseguramiento y acceso equitativo al sistema de salud.

El nicho de aplicabilidad de los seguros privados de salud alcanza a poco menos de un quinto de la población chilena (17\%), pero dado que los planes dependen en parte del monto de cotizaciones, la afiliación es más conveniente y con mayor cobertura de beneficios en los grupos de cotizantes con mayores ingresos o que aportan cotizaciones adicionales. Su crecimiento se ha favorecido por funcionar en un mercado poco regulado, donde prima el interés comercial por sobre las necesidades de salud de los beneficiarios. La capacidad de seguiros privados de comprar servicios alos proveedores asistenciales privados (escasos al inicio de la reforma), favoreció un importante crecimiento de las entidades de atención, incluyendola incorporación deprofesionales calificados que emigran del sector público. ${ }^{10-12}$

En contraste, el rol del Estado está disminuido en su capacidad de rectoría y rol regulatorio. La previsión pública de salud (FONASA) cuenta con financiamiento relativamente escaso respecto a su gran cobertura poblacional y necesidad de financiar la prestación del sistema público de salud, mientras que los seguros privados reciben subsidios estatales tanto directos como indirectos. Las condiciones laborales y salariales de la mayoría de la población son limitadas, y se produce una inequidad para los sectores de menos ingresos que se afilian a seguros privados, ya queademás de tener paquetes de menores beneficios, deben aportar mayor proporción de gastos en copago o de endeudamiento al momento de uso de servicios privados de salud..$^{10,12}$

En el mercado global mixto de aseguramiento, el sistema previsional público no está en condiciones de ofrecer alternativa competitiva de elección frente a los seguros privados de salud, por cuanto en su modalidad institucional permite atención a un sistema público asistencial con crisis financiera de larga data y bajo gran recarga de demanda, mientras que en su (pago del seguro público para atención privada(modalidad de libre elección) es de un aporte muy insuficiente al pago que debe hacer el beneficiario al momento de uso. ${ }^{8,10,11,14}$

\section{Consistencia de los seguros privados con el concepto de seguridad social}

Lo que se ha debatido respecto a los seguros privados de salud en su modalidad actual en Chile, es si efectivamente forman o no parte de la seguridad social. Su rol actual de discriminar por riesgo individual y cobrar una prima mayor a las personas que tienen más riesgo, es incompatible con participar en la captación de una cotización que es obligatoria. Si por el contrario, se considera que son parte de la seguridad social, entonces les corresponde participar de la recaudación de la cotización para salud, pero eso no les permitiría cobrar primas diferenciadas de acuerdo al riesgo que tengan las personas, porque una condición básica de la seguridad social es que desliga el riesgo 
de las personas individuales respecto de su capacidad de contribución financiera al sistema en su conjunto. ${ }^{15}$

Para que los seguros privados puedan ser consistentes con el concepto y rol de la seguridad social, requerirían incluir, entre otros aspectos un financiamiento que sea solidario entre sus afiliados bajo un concepto de derecho universal a la salud, que dicha afiliación sea abierta y no se discriminatoria por riesgo y capacidad de pago, que la protección sea vitalicia, y que los planes se fijen de modo colectivo (no por riesgo individual) y sus cálculos sean basados en estudios de proyecciones epidemiológicas, requerimiento de servicios de salud y costos de riesgos y requerimientos colectivos de recursos. La solidaridad incluye que en un fondo común se comparta contribuciones entre tienen mayores y menores recursos, jóvenes y viejos, sanos y enfermos. En la modalidad actual, los seguros privados de salud no cumplen con esos requisitos. ${ }^{10-12,16}$

\section{Logros y limitaciones de la recaudaciónde fondos}

Suficiencia de fondos recaudados: Los seguros privados tienen solvencia y adecuada recaudación de fondos desde la perspectiva empresarial, pero en general supera con creces la contribución obligatoria básica de 7\% de salarios, por recibiraportes adicionales de cotizantes (que mejoren el paquete de beneficios). Cuentan con multiplicidad de planes adaptados a capacidad de pago de prima, con distintos paquetes de beneficios en que se consideran edad y sexo, y condiciones preexistentes de salud al momento solicitar de afiliación. Tienen facultad relativa para ajuste unilateral de precios de planes (aunque sujeta a reclamos legales de cotizantes). ${ }^{10-12}$

Eficiencia en la recaudación: Cuentan con adecuados y detallados sistemas de registro, información y control, que les permiten conocer y controlar la recaudación que corresponda y los flujos del recurso financiero.

Sostenibilidad económica: Tienen solvencia económica y rentabilidad. Existen disposiciones legales para monitorear la solvencia de cada una y prevenir oportunamente el riesgo de bancarrota.

Equidad de las contribuciones: El sistema es inequitativo, pues la búsqueda de suficiencia de fondos pudiera entorpecerse si se utilizan criterios de equidad en las contribuciones de los afiliados. Por ese motivo, los seguros privados no contribuyen con la necesaria equidad que garantice el acceso universal a la atención de salud según necesidad, independiente de la capacidad de pago. ${ }^{17}$ Los empleadores no aportan contribuciones..${ }^{8,11}$ La clasificación de riesgos es individual, en circunstancias que debiera ser del grupo asegurado en su conjunto. ${ }^{15,17}$

Los afiliados no tienen información ni capacidad para negociar alternativas de beneficios, según derecho, necesidad y capacidad de pago. En los seguros abiertos al mercado, se excluye afiliar personas por motivos de discapacidad, riesgo o mala salud. ${ }^{17}$ Los planes discriminan contra mujeres, pues las mujeres tienden a recibir menor salario que los hombres, o están fuera del mercado lucrativo. Entonces tienden a quedar excluidas o bien reciben planes de salud con 


\section{$37 / /$}

menores beneficios y más caros. ${ }^{8,10}$

La discriminación de los seguros privados, especialmente al clasificar por riesgo, ha sido declarada inconstitucional y sujeta a multitud de reclamos legales de los cotizantes. ${ }^{17} \mathrm{~A} 1$ ser seguros privados competitivos, no existen mecanismos de compensación de riesgos entre aseguradoras, que contribuyan a regular el sistema privado de salud en términos de equidad. ${ }^{12,17}$

Solidaridad de contribución: Los seguros públicos y privados tienen lógicas distintas, en que los primeros usan un sistema de seguridad social de tipo Bismark y solidario, compartiendo recursos entre distintos riesgos, mientras que los seguros privados responden a planes individuales de contribuciones, lo que es incompatible con el concepto de solidaridad. Si un cotizante ya no puede costear la prima privada, le queda como alternativa la previsión pública (que incluso también cubre a los pobres). ${ }^{8,12}$ De acuerdo a las propias propuestas gubernamentales, se requiere de un plan garantizado común que permita contar con planes de beneficios solidarios y resguardo de copago excesivo por los servicios sanitarios, que incluya protección frente al riesgo financiero sobre el gasto catastrófico y el empobrecimiento. ${ }^{12,16}$

La solidaridad podría ser más facilitada con la creación de un sistema único de seguridad en salud (posición mayoritaria en la Comisión Asesora Presidencial de 2014), mientras que la existencia de multi-seguros requeriría una coordinación que en su conjunto permita un plan garantizado de salud que permita prestaciones universales, según necesidad. ${ }^{12}$

\section{Logros y limitaciones al aseguramiento del acceso económico}

Acceso al sistema de salud: La capacidad de pago a prestadores asistenciales privados contribuyó a un gran desarrollo del sistema asistencial privado, con encarecimiento de la atención de salud (a pesar que los seguros privados negocian contener costos) e inmigración de profesionales calificados desde sector público (el cual se mantiene históricamente en condiciones críticas de financiamiento y sobrecarga por demanda proveniente de más del $80 \%$ de la población). Eso repercute en menor acceso al sistema público (falta de capacidad asistencial y de acceso con largas listas de espera). ${ }^{10,12,14}$ Estos seguros determinan los copagos que corresponden a las personas para recibir intervenciones específicas, y préstamos a cotizantes (en actividades caras) en caso necesario, para asegurar el acceso adecuado.El acceso cubierto por los seguros privados es principalmente de servicios curativos, se requiere de cubrir promoción y prevención, así como establecer pertenencia con cobertura vitalicia de los cotizantes (es sólo temporal, mientras el cotizante paga prima). La cobertura debiera ser de otorgada por largo plazo a través de los diversos beneficios que se requieren a través del ciclo vital. ${ }^{12,17} \mathrm{El}$ acceso general a la atención de salud tiene a ser relativamente similar en sistemas privado y público, sin embargo el primero provee servicios de mayor calidad y especialidad. , $^{8,10-11}$ 
Equidad y eficiencia de una eventual mancomunación: No hay fondos mancomunados oalgún mecanismo de igualación de riesgos que pueda garantizar que la gente de los distintos planes pueda ser tratada por igual. Existe un fondo de compensación solidario entre ISAPRES respecto a un grupo de enfermedades garantizadas de atención. Los niveles de copago son diversos según los paquetes de beneficios que cada plan individual determina. ${ }^{12}$

\section{Logros y limitaciones del uso de los recursos}

Eficiencia en la utilización de los recursos: Existen adecuados mecanismos de compra y pago a los proveedores, lo que contribuye la calidad y la eficiencia de las prestaciones. Se realiza monitoreo y control de los beneficios y pagos realizados. Dicho control evita despilfarro y corrupción en flujo de dineros.Existen acuerdos institucionales y administrativos con prestadores para controlar y negociar pagos convenientes por las prestaciones definidas a los beneficiarios. Participan en las compras y contrataciones estratégicas para garantizar la mejor relación calidad-precio.Sin embargo, es necesario desarrollar un fondo mancomunado independiente, para la promoción y la prevención de la salud. Hay seguimiento periódico de los resultados y la eficiencia del uso de los fondos, así como reportes periódicos a la autoridad estatal rectora, que es la Superintendencia de Salud. ${ }^{10-12}$

Igualdad en la utilización de los recursos: Los paquetes de beneficios definidos para la prestación de servicios son individuales y al relacionarse con tarifas de aporte de prima según edad y sexo, resultan esencialmente inequitativos. ${ }^{12}$ Los Tribunales de Justicia cuestionan los fundamentos y la facultad asumida por ISAPRES de revisar los contratos y reajustar sus precios unilateralmente. ${ }^{12,17}$ Los cotizantes y beneficiarios de menos recursos, al tener menos capacidad de copago, acuden con mayor frecuencia al sistema asistencial público, lo que genera otra inequidad respecto a aquellos con mayores ingresos, paquetes de beneficios más amplios y mayor capacidad de copago..$^{10-12}$

Solidaridad de beneficios: El sistema de previsión privado no contribuye a la universalidad del sistema, que pueda considerar los mismos derechos en materia de salud, de toda lapoblación. ${ }^{12,15} \mathrm{La}$ perspectiva del derecho a la salud indica que se requiere crear una nueva institucionalidad que garantice, sin discriminación, el derecho al cuidado sanitario básico exigido por la justicia constitucional. ${ }^{17}$

Los beneficiarios del seguro privado cuentan con mayores ventajas de acceso al sistema asistencial privado que los beneficiarios del sistema previsional público (los que frecuentemente enfrentan largas listas de espera ante el sistema público de salud). Cuando un asegurado público accede al sistema privado, el seguro público aporta una baja proporción del costo de la prestación (especialmente en aquellas costosas). Por tanto el copago del asegurado público en la atención privada resulta mucho mayor y se convierte en un obstáculo financiero importante, especialmente cuando no cuenta con acceso oportuno al sistema público. ${ }^{10-12}$ Los seguros privados debieran 
garantizar beneficios equivalentes a aquellos definidos por el Estado para el sistema público (como existe para un grupo de enfermedades que legalmente se han determinado). ${ }^{10,11}$

\section{Cambios y reforma pendientes}

Propuestas gubernamentales: En 2010, una primera Comisión Asesora Presidencial sobre ISAPRES concentró sus propuestas en un plan garantizado de salud. En consecuencia, el gobierno presentó en 2011 un proyecto de ley al parlamento, que no prosperó. ${ }^{16}$ En 2014, se organizó una nueva Comisión Asesora Presidencial, entre cuyos puntos destaca que: (a) se requiere de un Plan de Seguridad Social (PSS) único y universal, es decir, al cual pueden acceder todos los habitantes del país;(b) se requiere establecer un Fondo Mancomunado Universal entre FONASA y las ISAPRES para romper la segmentación, introducir solidaridad inter-sistemas y permitir el financiamiento de prestaciones universales; (c) se requiere un fondo Inter-ISAPRES de compensación de riesgos para permitir la movilidad, el fin de la cautividad y pre-existencias, junto con introducir mayor solidaridad al sistema ISAPRES; y (d) se requiere crear una institución que administre un Fondo Subsidio de Incapacidad Laboral (SIL) de seguridad social con financiamiento tri-partito (Estado, empleadores y trabajadores) para terminar con los problemas para los trabajadores para obtener licencias médicas y su subsidio. ${ }^{12}$

Factibilidad de reformas: Los sistemas de seguros privados son resistentes al cambio, en resguardo de los intereses económicos y de poder.A ello contribuye que el Estado a través de gobierno y parlamento, cuenta con limitada factibilidad de usar mecanismos políticos para lograr los cambios y reformas que se necesitan en el seguro privado de salud. El país mantiene un sistema constitucional, legal y de constitución parlamentaria que tiende a proteger al sistema político y económico, contra cambios que pongan el riesgo al sistema neoliberal aún imperante. ${ }^{8,10-11}$

Las contribuciones aportadas por los miembros de las Comisiones Asesoras Presidenciales de 2010 y 2014,representa un consenso respecto a los aspectos generales y beneficios esperados de los seguros privados en el contexto de la protección social. Sin embargo, el aporte global de la propuesta es desprestigiado y obstaculizado por divergencias respecto a algunos de los mecanismos específicos que se pueden adoptar para perfeccionar el sistema y mejorar la regulación de los seguros privados. Las divergencias minoritarias surgen de del interés de proteger el aseguramiento y prestación privada, respecto a los riesgos financieros que involucrarían los cambios. En la divergencia minoritaria destacan el proponer que se disminuya el alcance esperado del fondo mancomunado de salud; no superar el 7\% de cotización obligatoria con la incorporación de adicional de una prima comunitaria; no terminar con la discriminación de quienes solicitan afiliación (que no se termine la obligación de declarar pre-existencia de enfermedad o discapacidad); y el derecho a que los seguros privados se integren verticalmente con un grupo de prestadores privados. ${ }^{12}$

Hay una asimetría de información ante la ciudadanía, dada la publicidad de los seguros privados y grupos de poder. La ciudadanía no está advertida de sus derechos, la inconsistencia de pagos, 
planes y beneficios que recibe, y falta mayor percepción de la protección social y la solidaridad involucrada. Ello refleja la necesidad de mayor conciencia, promoción, empoderamiento y presión social de la ciudadanía, en apoyo de los esfuerzos políticos gubernamentales que periódicamente se formulan para mejorar este sistema, mediante el logro de las reformas que se necesitan., ${ }^{2,12,17}$

\section{CONCLUSIÓN}

Creados a partir de 1981, estos seguros han logrado un sólido desarrollo institucional, con eficiente recaudación, uso y distribución de recursos, y contribuyen eficientemente al funcionamiento del sistema privado de salud. Sin embargo, no logran cumplir con los objetivos básicos de la seguridad social, en términos de equidad y solidaridad, y tienen otra lógica que el sistema público, lo que impide contribuir a un aseguramiento universal, equitativo y solidario, que a su vez contribuya al acceso universal al sistema de salud.

A pesar de su consolidación y solvencia, son económicamente vulnerables a cambios que arriesguen su rentabilidad, lo que dificulta contar con cambios y reformas conforme a derecho y necesidad de la población.

En su quehacer, predomina el interés comercial y sustentabilidad económica por sobre las necesidades y capacidad de pago de sus beneficiarios. Se requiere que adopten el principio de solidaridad, equidad, y contribuyan al acceso universal a la atención de salud, según necesidad e independiente de riesgos y capacidad de pago.

Entre otros mecanismos más específicos, se requiere un plan garantizado común a todos los tipos de seguros, que permita contar con planes de beneficios solidarios y resguardo de copago excesivo por los servicios sanitarios, que incluya protección frente al riesgo financiero sobre el gasto catastrófico y el empobrecimiento.

Si bien tienen limitaciones e inconsistencias en relación a la seguridad y protección social en salud, son la alternativa preferente de elección en el grupo de población con mayores ingresos, debido a la limitada y no competitiva opción que el seguro público puede ofrecer a este grupo.

Bajo mayor apoyo a la protección social desde 1990, los seguros privados han experimentado mejorías parciales y ajustes positivos, lo que aún es insuficiente. Se requiere de reformas concretaspara adecuar su rol a los principios de salud pública, pero ello se mantiene pendiente, debido a las restricciones que impone el modelo prevalente y la presión de los grupos de poder político y económico.

Es importante que la ciudadanía obtenga debida información sobre el aseguramiento y sus derechos, las brechas de cumplimiento en los seguros privados, que haya conciencia y empoderamiento sobre el tema, y que se apoye a los cambios y reformas que el Estado resuelva hacer, en beneficio de los derechos y necesidades de salud de la población. 


\section{REFERENCIAS BIBLIOGRÁFICAS}

1. Banco Mundial. Informe sobre el Desarrollo Mundial. Invertir en salud. Washington DC: BM; 1993. Disponible en: http://www-wds.worldbank.org/external/default/WDSContentServer/ WDSP/IB/2010/08/31/000333037_20100831011011/Rendered/PDF/121830WDR0SPANISH0Bo x35456B01PUBLIC1.pdf

2. OMS. Informe sobre la salud en el mundo: la financiación de los sistemas de salud: el camino hacia la cobertura universal. OMS: Ginebra, 2010. Disponible en: http://whqlibdoc.who. int/whr/2010/9789243564029_spa.pdf

3. Bazzani R., Eduardo Levcovitz E., Hernández A., Rodríguez O. y Rico de Sotelo C. Introducción. Nuevos horizontes para la producción de conocimiento y las políticas en protección social en salud, en Protección social en salud en América Latina y el Caribe. Investigación y políticas. Hernández A. y Rico de Sotelo C. (Ed.) IDRC 2011, pp. 11-32. Disponible en http:// www.idrc.ca/EN/Resources/Publications/openebooks/509-0/index.html

4. OPS/OMS-OIT, Ampliación de la protección social en materia de salud. Iniciativa conjunta de la Organización Panamericana de la Salud y la Organización Internacional del Trabajo, aprobada por la Resolución CSP26.R19 de Septiembre, 2002. Disponible en: http://www1.paho. org/spanish/gov/csp/csp26-12-s.pdf

5. OEA y OPS/OMS. La protección social en salud: avanzando hacia la cobertura universal de salud. Declaración conjunta de la Secretaría General de la Organización de los Estados Americanos y la Organización Panamericana de la Salud/Organización Mundial de la Salud. Disponible en: http://redproteccionsocial.org/sites/default/files/oea-paho_declaracion_conjunta_ final.pdf

6.Mejía-Ortega L., y Franco-Giraldo A. Protección social y modelos de desarrollo en América Latina Rev. Salud pública. 9 (3):471-483, 2007 Disponible en: http://www.scielosp.org/pdf/rsap/ v9n3/v9n3a16.pdf

7. Madies C., Chiarvetty S. y Chormy M. Aseguramiento y cobertura: dos temas críticos en las reformas del sector de la salud. Rev Panam Salud Publica/Pan Am J Public Health 8(1/2), 2000. Disponible en: http://www.scielosp.org/pdf/rpsp/v8n1-2/3002.pdf

8. Unger JP, De Paepe P, Solimano G. y Arteaga O. Chile's neoliberal health reform: An assessment and a critique. PLoS Medicine [Internet]. 2008 [citado 20 Feb 2010];5(4). Disponible en: http://www.plosmedicine. org/article/info:doi/10.1371/journal.pmed.0050079

9. Laurell AC, Herrera Ronquillo J. La Segunda Reforma de Salud. Aseguramiento y compraventa de servicios. Salud Colectiva. 2010:6(2):137-148. Disponible en: http://www.redalyc.org/ 
articulo.oa?id=73115020002

10. Urriola R. Chile: protección social de la salud. Rev Panam Salud Publica/Pan Am J Public Health 20(4), 2006. Disponible en: http://www.medwave.cl/medios/eventos/economiasalud/ docdescarga/ProtecionSocialdelaSalud12-2006.pdf

11. Mesa-Lagos C. Protección social en Chile: reformas para mejorar la equidad. Revista Internacional del trabajo, vol. 127 (2008), núm. 4 Disponible en: http://onlinelibrary.wiley.com/ doi/10.1111/j.1564-9148.2008.00041.x/pdf

12. Gobierno de Chile. Informe de la Comisión Asesora Presidencial para el Estudio y Propuesta de un Nuevo Marco Jurídico para el Sistema Privado de Salud. Chile, Octubre de 2014. Disponible en: http://web.minsal.cl/sites/default/files/Informe\%20Final\%20 Comisi\%C3\%B3n\%20Asesora\%20Presidencial.pdf

13. Gattini C. et al. Salud en Chile 2010. Panorama de la situación de salud y del sistema de salud en Chile. Oficina de OPS/OMS en Chile, Santiago: 2011 Disponible en: http://new.paho. org/chi/images/PDFs/salud\%20chile\%202010.pdf

14. Gattini et al. Panorama y tendencias de la salud en Chile. Revisión 2013. Observatorio Chileno de Salud Pública (OCHISAP) Escuela de salud Pública Fac. Medicina Universidad de Chile. Disponible en: http://www.ochisap.cl/images/Panorama\%20y\%20Tendencias\%20de\%20 la\%20Salud\%20en\%20Chile\%20Revision\%202013.pdf

15. Arteaga O. Tendencias en la organización de sistemas y servicios de salud. Presentación a la Academia Chilena de Medicina, 3 de julio de 2013. Instituto de Chile. Disponible en http:// www.institutodechile.cl/2012/web/post.php?id=102

16. Ministerio Secretaría General de la Presidencia. "Informe de Comisión de Expertos para Creación de Plan Garantizado de Salud en las Isapres"en Informes de las Comisiones Asesoras Presidenciales 2010-2014. Gobierno del Presidente Sebastián Piñera,pp. 575652, Marzo 2014. Disponible en: http://gestion2010-2014.cumplimiento.gob.cl/wp-content/ uploads/2014/03/140319_informes_comisiones_asesoras_presidenciales_2010-2014.pdf

17 Zúñiga A. Propuesta para la reforma definitiva del sistema privado de salud chileno Rev. Méd. Chile 2014; 142:501-505. Disponible en: http://gestion2010-2014.cumplimiento.gob.cl/wpcontent/uploads/2014/03/140319_informes_comisiones_asesoras_presidenciales_2010-2014.pdf

Artigo apresentado em 30-07-15

Artigo aprovado em 25-02-16

Artigo publicado no sistema em 30-03-16 